\title{
Modelamiento de la irradiancia y la temperatura ambiente utilizando funciones de probabilidad
}

\section{Irradiation and ambient-temperature model using probability functions}

\section{Johann Alexander Hernández Mora}

Ingeniero Electricista, magíster en Ingeniería Eléctrica, Doctor en Ingeniería Eléctrica. Docente asistente de la Universidad Distrital Francisco José de Caldas. Bogotá, Colombia. Contacto: jahernandezm@udistrital.edu.co

\section{César Leonardo Trujillo Rodríguez}

Ingeniero Electrónico, magíster en Ingeniería Eléctrica, Doctor en Ingeniería Electrónica. Docente asociado de la Universidad Distrital Francisco José de Caldas. Bogotá, Colombia. Contacto: cltrujillo@udistrital.edu.co

\section{William Andrés Vallejo Lozada}

Químico, Doctor en Química. Docente de la Universidad del Atlántico. Barranquilla, Colombia. Contacto: williamvallejo@mail.uniatlantico.edu.co

Palabras clave: distribución de Weibull, función de probabilidad, irradiancia, temperatura.

Keywords: Weibull distribution, function probability, radiation, temperature.

\section{RESUMEN}

En este trabajo se desarrolló una metodología estadística para caracterizar la irradiancia solar y temperatura ambiente a partir de medidas reales. Estas variables determinan el comportamiento de la energía solar en un lugar determinado y son fundamentales para establecer el desempeño de diversos sistemas que usan energía solar como el caso de los sistemas fotovoltaicos o los solares térmicos. Debido a que estos parámetros no son controlables por el hombre, ya que se caracterizan por tener un comportamiento aleatorio en la superficie terrestre, se desarrolló una metodología para obtener una relación matemática que en primera instancia determina una función 
de distribución de probabilidad para 12 horas al día, de 6 de la mañana (6:00 a.m.) a 6 de la tarde (6:00 p.m.), con el fin de predecir el comportamiento de estas variables meteorológicas. Con estas funciones de densidad, se calculan luego las respectivas funciones de probabilidad acumuladas. En los casos en que estas no se pueden determinar de forma determinística, se calcula numéricamente un polinomio que mejor las representa. Dichas expresiones obtenidas, sirven como base para realizar predicciones estadísticamente confiables del desempeño de distintos sistemas que utilicen como fuente primaria la energía solar, utilizando métodos estocásticos, como por ejemplo las simulaciones de Montecarlo. Para mostrar la metodología descrita, se tomaron como ejemplo datos medidos en la ciudad de Bogotá. Finalmente, se presentan las respectivas expresiones que caracterizan la irradiancia y la temperatura ambiente para cada hora a partir de un número aleatorio entre 0 y 1 de manera estadísticamente confiable.

\section{ABSTRACT}

In this work, we develop a statistical methodology to characterize solar radiation and ambient temperature from real measurements. These parame- ters determine the behavior of solar energy in a particular location and they are essential to establish the performance of different systems that use this type of energy, such as photovoltaic or solarthermal systems. Since these ambient parameters have a random behavior and they cannot be controlled by human intervention over the earth's surface, a methodology to obtain a probability density distribution for twelve (12) hours a day (from 6:00 a.m to $6: 00$ p.m.) was built so as to predict solar energy behavior. From these density functions, corresponding cumulative probability functions are calculated. In the cases where variables cannot be deterministically determined, a numerical-best polynomial representation is found. The cumulative probability functions obtained in this work can be used as a basis to constructing statically reliable predictions of performance for different solar energy-based systems. These systems may serve as a first energy resource by using stochastic methods like Montecarlo simulations. As an example, in this paper we take data from measurement campaigns conducted in Bogotá to explain the aforementioned methodology. Finally, expressions that characterize the corresponding radiation and ambient temperature for each hour (from a statistically reliable random number between 0 and 1) are shown.

\section{INTRODUCCIÓN}

El agotamiento de los recursos combustibles fósiles y la contaminación producida por uso excesivo de estos, se ha convertido en un problema mundial debido a que su uso ha provocado el deterioro progresivo de la capa de ozono y es una de las principales razones del calentamiento global. Por esto se genera la necesidad de buscar fuentes de energía limpias, y en la actualidad las energías renovables que utilizan la energía solar como fuente primaria de energía (como la energía

solar foto-voltaica o térmica) son prometedoras, debido a que el sol es una fuente inagotable de energía. La disponibilidad del recurso es alta, ya que los rayos solares que llegan a la superficie terrestre pueden satisfacer más de 10000 veces el consumo de energía mundial, y adicionalmente las emisiones de $\mathrm{CO}_{2}$ pueden ser reducidas significativamente utilizando este tipo de tecnologías. Un sistema solar autónomo que sustituya a un grupo electrógeno diesel típico reducirá alrededor de $1 \mathrm{~kg}$ de $\mathrm{CO}_{2}$ por $\mathrm{kW} / \mathrm{h}$ producido. Por su parte, los sistemas FV conectados a la red disminui- 
rán $\mathrm{CO}_{2}$ dependiendo del perfil de producción de electricidad existente en distintos países. La cifra media mundial se ha establecido en $0,6 \mathrm{~kg}$ de $\mathrm{CO}_{2}$ por $\mathrm{kW} / \mathrm{h}[1]$.

Uno de los parámetros más importantes en el diseño de sistemas FV es el recurso solar disponible. Desafortunadamente la irradiancia solar se caracteriza por tener un comportamiento impredecible sobre la superficie terrestre debido a que es afectada por los fenómenos meteorológicos (lluvias, nubes, vientos, etc.) en su trayectoria desde la parte exterior de la atmósfera. En la actualidad existen diferentes programas que simulan el posible comportamiento de la irradiancia y la temperatura ambiente en diferentes partes del planeta. Sin embargo, debido a las características atmosféricas y geológicas propias de cada lugar estos simuladores muchas veces se alejan de la realidad debido a que se basan principalmente en cálculos astronómicos. Se hace necesario el desarrollo de herramientas que permitan obtener información acerca del comportamiento de la irradiancia solar para partes específicas del globo. En este trabajo se desarrolló una metodología que caracteriza el comportamiento real de la iradiancia y la temperatura ambiente, utilizando datos reales suministrados por el grupo de materiales semiconductores y energía solar (GMS\&ES) de la Universidad Nacional de Colombia [2]. A partir de las bases de datos reales, se obtuvo una distribución probabilística cada hora medida, durante las horas en que hubo presencia del sol. Para el caso de Bogotá, se tomaron las horas comprendidas entre las 6 de la mañana y las 6 de la tarde.

\section{METODOLOGÍA Y RESULTADOS}

En el presente capítulo se describe la metodología desarrollada mediante 2 fases: obtención de funciones de densidad de probabilidad y obtención de funciones de probabilidad acumulada. Esta última se realiza de forma determinística o numérica según sea el caso. En cada paso se van mostrando los resultados obtenidos.

\subsection{Obtención de las funciones de densidad de probabilidad}

El primer paso naturalmente es contar con una base de datos donde se tengan medidas de cada una de las variables. Para el caso particular, el GMS\&ES tiene una estación meteorológica [3] que contiene una base de datos recolectados desde el 2003 para irradiancia, y desde el 2005 para temperatura. En la tabla 1 se muestra tasa de muestreo para la temperatura ambiente y la irradiancia solar.

Para la irradiancia se tomaron los datos de los 7 años mostrados en la tabla 1, lo que representa un total de 2557 días. Sin embargo, debido a diferentes eventos ocurridos con el sistema de adquisición de datos (cortes de fluido eléctrico, calibración de instrumentos, etc.) no se dispone de información de todos los días del periodo de muestreo. La cantidad de días de los cuales se tiene información completa es de 2203, cifra que equivale al $86,16 \%$ de los días bajo estudio. Para el caso de la temperatura ambiente, se tiene información completa de 1583 días del total de 1826 , que corresponde al $86,69 \%$.

Una vez se conoce la cantidad de datos totales para cada variable, estos son procesados con el fin de obtener una función de densidad de probabilidad que mejor se ajuste a los datos reales. En este procesamiento de los datos se utilizó el siguiente procedimiento:

Tabla 1. Tasas de muestreo de datos por hora de los parámetros atmosféricos estudiados

\begin{tabular}{|c|c|c|}
\hline Año & Irradiancia & Temperatura \\
\hline $2003-2005$ & 120 & 120 \\
\hline $2006-2009$ & 60 & 60 \\
\hline
\end{tabular}

Nota: las medidas de temperatura inician el 2005.

Fuente: elaboración propia. 


\section{investigación}

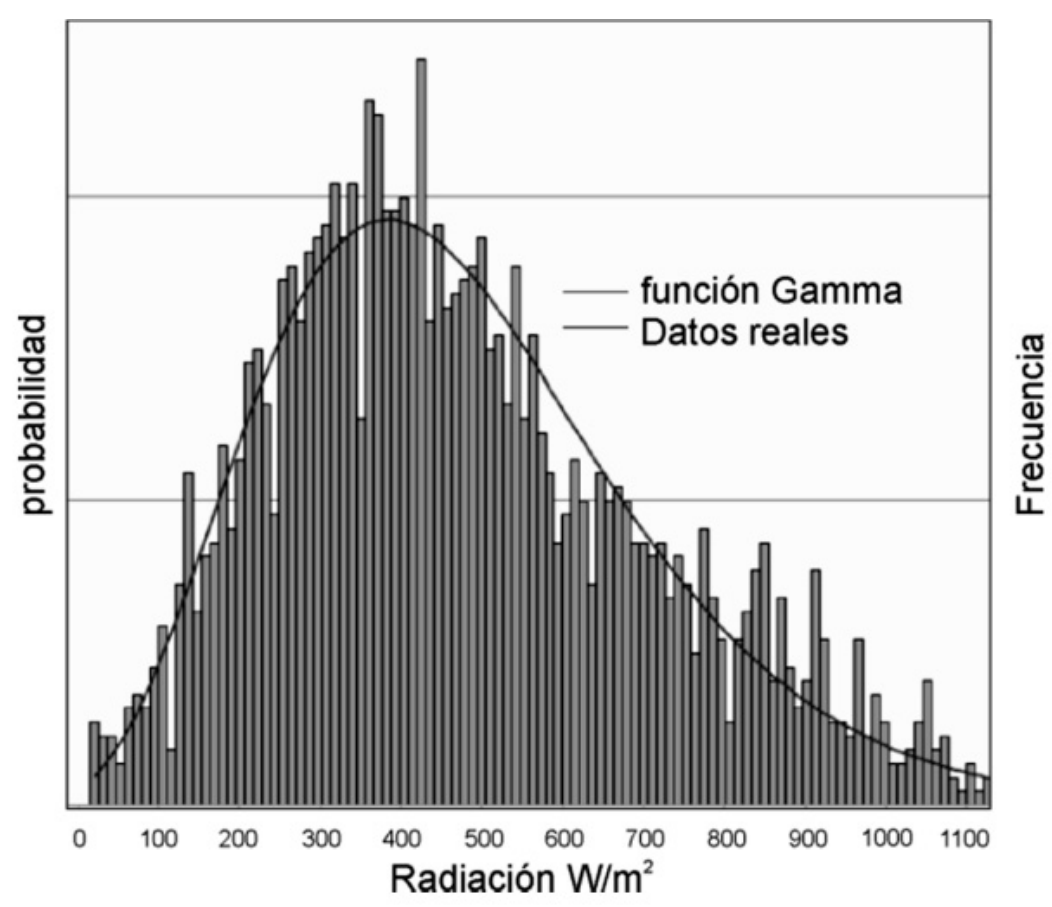

Figura 1. Función de densidad de probabilidad para la irradiancia a las $12 \mathrm{~m}$

Fuente: elaboración propia

- Se hicieron los promedios por hora 6 de la mañana a 6 de la tarde, teniendo en cuenta la tasa de muestreo por hora para cada año.

- A los datos obtenidos por hora, se les realizó la prueba de Anderson-Darling [4], [5], prueba no paramétrica que determina a qué distribución de probabilidad se asemejan los datos recolectados. Esta prueba fue realizada con la ayuda de la herramienta computacional llamada "CrystalBall”®.

- Estas funciones de densidad de probabilidad que caracterizaban cada hora, se determinaron por medio del Coeficiente A-D: si este coeficiente es menor a 10, la función de probabilidad encontrada representa de manera confiable el comportamiento de la variable aleatoria [4], [5], y entre menor sea el valor de este coeficiente, mejor es la representación de la función de densidad de probabilidad.
La figura 1 muestra la función de densidad de probabilidad que mejor se ajusta para el caso de la irradiancia a las $12 \mathrm{pm}$. Los parámetros de la función obtenida por el software se pueden apreciar en la tabla 2. Se puede verificar que varias funciones tienen el coeficiente A-D menor a 10, pero la función gamma es la función que mejor representa los datos de irradiancia de la $12 \mathrm{~m}$, ya que tiene el valor más cercano a cero.

El procedimiento anteriormente descrito se repitió para las demás horas comprendidas entre el rango de 7 de la mañana a 6 de la tarde.

La tabla 3 muestra las funciones que mejor ajustan la irradiancia y la temperatura ambiente (de acuerdo con el criterio del menor coeficiente A-D) para cada una de las horas.

Con esta metodología, la cual puede ser utilizada en cualquier lugar donde se tengan datos previa- 
Tabla 2. Parámetros de la función de densidad de probabilidad para la irradiancia a $12 \mathrm{pm}$

\begin{tabular}{|l|c|}
\hline \multicolumn{1}{|c|}{ Función } & Parámetro A-D \\
\hline Gamma & 1,3905 \\
\hline Logarítmica & 1,7498 \\
\hline Weibull & 1,7835 \\
\hline Beta & 2,8630 \\
\hline Logística & 15,2927 \\
\hline Triangular & 32,4929 \\
\hline t-student & 54,0263 \\
\hline Exponencial & 251,3276 \\
\hline
\end{tabular}

Fuente: elaboración propia.

mente recolectados, se caracteriza el comportamiento de estas variables.

\subsection{Obtención de las funciones acumuladas}

Debido a que los sistemas solares fotovoltaicos dependen de la irradiancia solar y la temperatura, variables que no son $100 \%$ predecibles, no es posible desarrollar un estudio en forma determinís- tica de su comportamiento; solo podemos utilizar métodos probabilísticos. Existen diversos métodos no determinísticos, como por ejemplo el de Monte Carlo. Este emula el funcionamiento de un sistema considerando variables aleatorias que están caracterizadas por funciones de probabilidad acumuladas, que se obtienen de forma analítica a partir de las funciones de densidad probabilidad, con el fin de llegar a una expresión donde la variable aleatoria (en este caso, la irradiancia o la temperatura) esté en función de la probabilidad [6]. Estas variables aleatorias se deben modelar por medio de funciones de distribución acumuladas que indiquen el valor de dicho parámetro a partir de números aleatorios que generalmente toman valores entre cero y uno. Aunque anteriormente se determinaron estas funciones de densidad de probabilidad para cada hora. La mayoría de estas funciones no permiten determinar de forma analítica el comportamiento de estos parámetros atmosféricos, ya que se necesita primero encontrar la distribución acumulada (la cual para algunas ni siquiera está definida), y luego despejar el parámetro dejando como variable indepen-

Tabla 3. Funciones de densidad probabilidad para la irradiancia y la temperatura para las horas con radiación solar

\begin{tabular}{|c|l|c|c|c|}
\hline \multirow{2}{*}{ Hora } & \multicolumn{2}{|c|}{ Irradiancia } & \multicolumn{2}{c|}{ Temperatura } \\
\cline { 2 - 5 } & Ajuste & Coeficiente A-D & Ajuste & Coeficiente A-D \\
\hline $6-7$ & Log normal & 2,0378 & Logistic & 0,6374 \\
\hline $7-8$ & Gamma & 0,7752 & Gamma & 0,8609 \\
\hline $8-9$ & Weibull & 2,9365 & Weibull & 1,8511 \\
\hline $9-10$ & Beta & 4,172 & Logistic & 1,9373 \\
\hline $10-11$ & Max extreme & 4,9255 & Weibull & 1,7746 \\
\hline $11-12$ & Max Extreme & 3,0006 & Weibull & 2,4066 \\
\hline $12-1$ & Gamma & 1,3905 & Weibull & 1,4208 \\
\hline $1-2$ & Gamma & 1,0283 & Weibull & 0,8829 \\
\hline $2-3$ & Beta & 0,6386 & Weibull & 0,5678 \\
\hline $3-4$ & Weibull & 1,9045 & Weibull & 0,5307 \\
\hline $4-5$ & Weibull & 1,0772 & Weibull & 1,9689 \\
\hline $5-6$ & Log normal & 0,5334 & Weibull & \\
\hline
\end{tabular}

Fuente: elaboración propia. 


\section{investigación}

diente la distribución acumulada. De las funciones seleccionadas, únicamente la función Weibull permite hacer dicho despeje analítico, ya que su función de densidad de probabilidad está representada por:

$$
\mathrm{f}(\mathrm{x})=\frac{\beta}{\eta}\left(\frac{x-x_{0}}{\eta}\right)^{\beta-1} \exp \left[-\left(\frac{x-x_{0}}{\eta}\right)^{\beta}\right]
$$

Y la función de distribución acumulada es:

$$
F(x)=1-\exp \left[-\left(\frac{x-x_{0}}{\eta}\right)^{\beta}\right]
$$

Las ecuaciones (1) y (2) solo se aplican para valores de $(x-x 0) \geq 0$. Para valores de $(x-x 0)<0$, las funciones de densidad toman un valor de cero. Las constantes que aparecen en las expresiones anteriores tienen la siguiente interpretación física:

- $x_{0}$ es el parámetro de ubicación o vida mínima y define el punto de partida u origen de la distribución.

- $\eta$ es el factor de escala, extensión de la distribución a lo largo del eje de las abscisas.

- $\beta$ es el factor de forma y representa la pendiente de la recta describiendo el grado de variación de la distribución.

Despejando el parámetro a determinar (x) se encuentra la siguiente relación:

$$
\mathrm{x}=x_{0}+\eta\left[[-\ln (1-F(x))]^{\frac{1}{\beta}}\right]
$$

Para la distribución Weibull a partir de un número aleatorio $\mathrm{F}(\mathrm{x})$ entre 0 y 1.

Para las demás distribuciones que no permiten realizar un despeje analítico como el de la función Weibull, se realiza el siguiente procedimiento:

- Una vez realizado el ajuste para cada hora con el programa Crystal Ball ${ }^{\circledR}$, se utiliza el reporte que este produce. En este reporte se generan 2 pares de vectores de 100 datos cada uno, donde se representa la función de densidad de probabilidad o la función acumulada de probabilidad según el usuario lo requiera.

- Con los pares de vectores que representan la función acumulada de probabilidad, y realizando un cambio de ejes, se utiliza el lenguaje de programación TableCurve ${ }^{\circledR}$, para encontrar el polinomio que mejor ajusta la relación entre dicho par de vectores, lo que da como resultado una relación entre el parámetro buscado (irradiancia o temperatura ambiente) en función de su probabilidad (número aleatorio entre cero y uno). La figura 2 muestra el ejemplo de dicho ajuste para el caso de la temperatura de las 6 de la mañana a las 6 de la tarde.

La tablas 4 y 5 resumen las relaciones matemáticas para determinar la irradiancia solar y la temperatura ambiente a partir de la generación de números aleatorios entre cero y uno, de acuerdo con las distribuciones de probabilidad acumulada, obtenidas a partir de los datos reales suministrados.

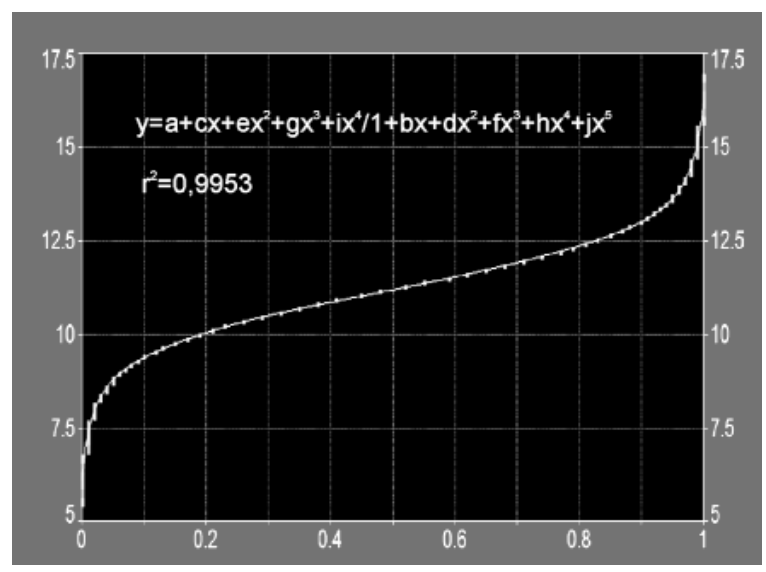

Figura 2. Ajuste en TableCurve ${ }^{\circledR}$ para determinar la temperatura de las 6 a.m. en función de un número aleatorio entre 0 y 1 a partir de su función de probabilidad (log Normal para este caso)

Fuente: elaboración propia. 


\section{investigación}

Tabla 4. Relaciones matemáticas para determinar la irradiancia a partir de un número aleatorio entre cero y uno

\begin{tabular}{|c|c|c|}
\hline Hora & Relación & $\begin{array}{l}\text { Coeficiente } \\
\text { de relación } \\
\text { (R) }\end{array}$ \\
\hline 6-7 & $\mathrm{R}=\frac{4,447+620 \mathrm{p}+3930,84 p^{2}-1067,17 p^{3}-3112,63 p^{4}}{1+34,53 \mathrm{p}+105,744 p^{2}-219,4787 p^{3}+79,9 p^{4}}$ & 0,98047241 \\
\hline 7-8 & $\mathrm{R}=\frac{1,8729+5405,21 \mathrm{p}+93201,76 p^{2}-20448,4 p^{3}-67255,8856 p^{4}}{1+105,566 \mathrm{p}+656,64514 p^{2}-922,0077 p^{3}+183,8887 p^{4}}$ & 0,99761671 \\
\hline 8-9 & $\mathrm{R}=6+341\left[[-\ln (1-p)]^{\frac{1}{2,55285}}\right]$ & $\begin{array}{l}\text { N/A: Función } \\
\text { Weibull }\end{array}$ \\
\hline $9-10$ & $\mathrm{R}=\frac{76,47+9393,53 \mathrm{p}+71803,53 p^{2}-20397,81 p^{3}-56070,4264 p^{4}}{1+62,7626 \mathrm{p}+137,2124 p^{2}-174,192 p^{3}-102,3366 p^{4}+79,658 p^{5}}$ & 0,99791445 \\
\hline 10-11 & $\mathrm{R}=\frac{48,50+8909,9 \mathrm{p}+172080,2 p^{2}+32332,46 p^{3}-188002,156 p^{4}}{1+57,574 \mathrm{p}+585,3165 p^{2}-753,396 p^{3}+127,8 p^{4}}$ & 0,99535313 \\
\hline 11-12 & $\begin{array}{l}\mathrm{R}=36,04+4139,87 \mathrm{p}-33376,4 p^{2}+83632,44 p^{3}+608063,77 p^{4} \\
-5,1563 e 6 \mathrm{p}^{5}+1,6835 e 7 p^{6}-2,98 e 7 p^{7}+3,01 e 7 p^{8}-1,6272 e 7 p^{9} \\
+3,66408 e 6 p^{10}\end{array}$ & 0,9954401 \\
\hline $12-1$ & $\mathrm{R}=\frac{-2,9+11500,29 \mathrm{p}+198323,2 p^{2}-95094,9 p^{3}-96526,2574 p^{4}}{1+104,7 \mathrm{p}+442,83 p^{2}-749,058 p^{3}+214,46 p^{4}}$ & 0,99714875 \\
\hline $1-2$ & $\begin{array}{l}\mathrm{R}=-16,98+4790,738 \mathrm{p}-57985,5641 p^{2}+402299,3 p^{3}-1,4842 \mathrm{e} 6 p^{4} \\
+2,8001 e 6 \mathrm{p}^{5}-1,7193 e 6 p^{6}-2,9126 e 6 p^{7}+6,41642 e 6 p^{8} \\
-4,7096 e 6 p^{9}+1,26122 e 6 p^{10}\end{array}$ & 0,9972859 \\
\hline $2-3$ & $\mathrm{R}=\frac{12,3252+1316,495 \mathrm{p}-1005,42 p^{2}-282,086 p^{3}}{1+2,9476 \mathrm{p}-6,4882 p^{2}+2,582 p^{3}}$ & 0,99667552 \\
\hline $3-4$ & $\mathrm{R}=-0,923+296,2\left[[-\ln (1-p)] \frac{1}{1,7178}\right]$ & $\begin{array}{l}\text { N/A: Función } \\
\text { Weibull }\end{array}$ \\
\hline $4-5$ & $\mathrm{R}=168\left[[-\ln (1-p)] \frac{1}{1,58451}\right]$ & $\begin{array}{l}\text { N/A: Función } \\
\text { Weibull }\end{array}$ \\
\hline $5-6$ & $\mathrm{R}=\frac{0,48268+442,48 \mathrm{p}+2939,3 p^{2}+4211,585 p^{3}-6981,77 p^{4}}{1+39,528 \mathrm{p}+165,915 p^{2}-320,585 p^{3}+116,13 p^{4}}$ & 0,96157728 \\
\hline
\end{tabular}

Fuente: elaboración propia.

134 Tecnura | Vol. $18 \mid$ No. 39 | enero - marzo de 2014 


\section{investigación}

Tabla 5. Relaciones matemáticas para determinar la temperatura ambiente a partir de un número aleatorio entre cero y uno

\begin{tabular}{|c|c|c|}
\hline Hora & Relación & $\begin{array}{l}\text { Coeficiente } \\
\text { de correlación }\end{array}$ \\
\hline 6-7 & $\mathrm{R}=\frac{6,116+424,48 \mathrm{p}-2459,14 p^{2}+7907,5 p^{3}-5768,423 p^{4}}{1+41,234 \mathrm{p}-257,759 p^{2}+864,467 p^{3}-783,81 p^{4}+141,6496 p^{5}}$ & 0,9953649 \\
\hline $7-8$ & $\mathrm{R}=\frac{9,17+860,51 \mathrm{p}-1022,69 p^{2}-1861,67 p^{3}+3320,263 p^{4}-1303,97 p^{5}}{1+74,07 \mathrm{p}-134,58865 p^{2}-33,8655 p^{3}+171,223 p^{4}-77,8264 p^{5}}$ & 0,99632323 \\
\hline 8-9 & $\mathrm{R}=9,14+7,63\left[[-\ln (1-p)]^{\frac{1}{4,08594}}\right]$ & $\begin{array}{l}\text { N/A: Función } \\
\text { Weibull }\end{array}$ \\
\hline $9-10$ & $\mathrm{R}=\frac{10,64+680,49 \mathrm{p}-3568,39 p^{2}+9865,43 p^{3}-6867,257 p^{4}}{1+42,278 \mathrm{p}-238,3 p^{2}+688,64 p^{3}-585,3 p^{4}+96,682 p^{5}}$ & 0,99536474 \\
\hline $10-11$ & $\mathrm{R}=7,73+11,29\left[[-\ln (1-p)]^{\frac{1}{6,14}}\right.$ & $\begin{array}{l}\text { N/A: Función } \\
\text { Weibull }\end{array}$ \\
\hline $11-12$ & $\mathrm{R}=7,36+12,05\left[[-\ln (1-p)] \frac{1}{6,13812}\right.$ & $\begin{array}{l}\text { N/A: Función } \\
\text { Weibull }\end{array}$ \\
\hline $12-1$ & $\mathrm{R}=7+12,40\left[[-\ln (1-p)]^{\frac{1}{5,96153}}\right]$ & $\begin{array}{l}\text { N/A: Función } \\
\text { Weibull }\end{array}$ \\
\hline $1-2$ & $\mathrm{R}=4,24+14,96\left[[-\ln (1-p)] \frac{1}{6,64411}\right]$ & $\begin{array}{l}\text { N/A: Función } \\
\text { Weibull }\end{array}$ \\
\hline $2-3$ & $R=3.71+14.98\left[[-\ln (1-p)] \frac{1}{6.21306}\right]$ & $\begin{array}{l}\text { N/A: Función } \\
\text { Weibull }\end{array}$ \\
\hline $3-4$ & $R=6.2+11.65\left[[-\ln (1-p)]^{\frac{1}{4.7535}}\right.$ & $\begin{array}{l}\text { N/A: Función } \\
\text { Weibull }\end{array}$ \\
\hline 4-5 & $\mathrm{R}=7,38+9,16\left[[-\ln (1-p)]^{\frac{1}{4,23}}\right]$ & $\begin{array}{l}\text { N/A: Función } \\
\text { Weibull }\end{array}$ \\
\hline $5-6$ & $\mathrm{R}=7,38+7,25\left[[-\ln (1-p)] \frac{1}{5,20}\right.$ & $\begin{array}{l}\text { N/A: Función } \\
\text { Weibull }\end{array}$ \\
\hline
\end{tabular}

Fuente: elaboración propia. 
Es importante determinar el coeficiente de correlación para las expresiones obtenidas. En nuestro estudio, todos los casos arrojaron un resultado mayor a 0,95 , lo que garantiza que los resultados son confiables, es decir, los polinomios caracterizan de la mejor forma los datos históricos de irradiancia y temperatura.

Una vez establecida la forma de determinar los polinomios para cada una de las horas de las variables meteorológicas, tendremos accesos a valores confiables de irradiancia y temperatura.Estos valores se pueden utilizar como parámetros de entrada para realizar predicciones del comportamiento de sistemas fotovoltaicos en redes eléctricas. Las simulaciones de Monte Carlo pueden ser utilizadas para realizar estas predicciones. En estas simulaciones, el sistema es dividido en elementos cuyo comportamiento se puede predecir ya sea determinísticamente o mediante distribuciones de probabilidad. Estos elementos son luego combinados para determinar el comportamiento general del sistema. Dada su naturaleza estocástica, la simulación de Monte Carlo no alcanza los niveles de precisión de las técnicas analíticas en sus resultados, sino que solo entrega estimaciones de los resultados exactos. Sin embargo, la resolución de los problemas formulados mediante este método es más simple, constituyendo la única opción adecuada para muchos sistemas eléctricos reales.

\section{CONCLUSIONES}

Los sistemas que utilizan la energía solar como fuente primaria para transformarla en otro tipo de energía, como los solares térmicos o los fotovoltaicos, presentan el inconveniente de que los parámetros meteorológicos que la caracterizan (irradiancia y temperatura ambiente) presentan un comportamiento aleatorio fuera del control humano, lo que hace que una predicción del funcionamiento de estos sistemas no pueda hacerse de manera determinística.

La metodología desarrollada en el presente trabajo permite determinar de una manera sencilla las funciones de densidad probabilidad que representen de forma estadísticamente confiable el comportamiento de la irradiancia y la temperatura ambiente a partir de mediciones reales. Se recomienda tener una base de datos de por lo menos 5 años.

Como es altamente probable que dichas funciones de densidad de probabilidad no permitan determinar de manera analítica la respectiva función acumulada o el despeje de la variable aleatoria de esta respectiva función acumulada, la metodología propuesta permite también determinar polinomios estadísticamente confiables que superan el inconveniente de realizar este despeje. 


\section{REFERENCIAS}

[1] Renewable Energy Policy Network for the 21st Century, REN21. Report 2011, [en línea]. Disponible en www.ren21.net/ publications/default.asp.

[2] N. Forero, J. Hernández and G. Gordillo."Development of a monitoring system for a PV solar plant”, Energy Conversion and Management, vol. 47, Issues 15-16, pp. 2329-2336, 2006.

[3] T.W. Anderson, and D.A. Darling, "A test of Goodness of Fit", Journal of the American Statistical Association, vol. 49, pp. 765-769, 1954.
[4] T.W. Anderson, and D.A. Darling, “Asymptotic theory of certain Goodness of fit criteria based on stochastic processes", Annals of Mathematical Statistics, vol. 23, pp. 193212, 1952.

[5] A. Torres M., Probabilidad, variables aleatorias, confiabilidad y procesos estocásticos, Universidad de los Andes, 2002.

[6] De Vos, A. Thermodynamics of radiation energy conversion in one and in three physical dimensions. Journal of Physics and Chemistry of Solids, vol. 49, Issue 6, 1988, pp.725730 . 